\title{
Towards an Explanation of the Mesoscopic Double-Slit Experiment, a new model for charging of a Quantum Dot
}

\author{
P.G.Silvestrov ${ }^{1,2}$ and Y.Imry ${ }^{2}$ \\ ${ }^{1}$ Budker Institute of Nuclear Physics, 630090 Novosibirsk, Russia, \\ ${ }^{2}$ Weizmann Institute of Science, Rehovot 76100, Israel
}

For a quantum dot (QD) in the intermediate regime between integrable and fully chaotic, the widths of singleparticle levels naturally differ by orders of magnitude. In particular, the width of one strongly coupled level may be larger than the spacing between other, very narrow, levels. In this case many consecutive Coulomb blockade peaks are due to occupation of the same broad level. Between the peaks the electron jumps from this level to one of the narrow levels and the transmission through the dot at the next resonance essentially repeats that at the previous one. This offers a natural explanation to the recently observed behavior of the transmission phase in an interferometer with a QD.

PACS numbers: 73.23.Hk, 05.45.-a, 73.20.Dx

In spite of much progress in the fabrication and experimental investigation of ultrasmall few-electron devices such as Quantum dots [1], many experimentally observed features of these systems still remain unexplained.

A challenging problem which has resisted adequate theoretical interpretation arises from the experiment [2] which determines the phase of the wave transmitted through the QD [3]. The main goal of this paper will be to find a mechanism which may lead to a satisfactory explanation of these results. Hopefully our approach will also allow to shed some light on other open problems concerning the Coulomb blockade (CB) 《4 in QD-s.

In the experiment of ref. [2], in addition to the conductance of the QD, the phase of the electron transmitted through the QD was measured via an interference arrangement. In accordance with the Breit-Wigner picture, the phase increased by $\pi$ around each CB peak. Absolutely unexpected, however, was a fast jump of the phase by $-\pi$ between the resonances near the minimum of the transmitted current. Such a behavior is in evident contradiction with what one would expect if the transmission of the current proceeds via consecutive levels in 1-dimensional quantum well.

In a two-dimensional QD the phase drops associated with the nodes of the transmission amplitude arise already within the single-particle picture [5.6]. However, in order to have a sequence of such events one should consider a QD of a very special form. The model of ref. [7] also does not allow to explain the series of drops. The mechanism of refs. [8,9] makes nontrivial assumptions on the geometry of the QD and the way it changes under the change of plunger gate voltage. An interesting generic mechanism suggested recently in ref. 10] may indeed lead to the correlations in transmission at many consecutive valleys, but the predicted phase behavior differs from what has been seen experimentally.

In this paper we propose a mechanism according to which the transmission at many $\mathrm{CB}$ peaks proceeds through one and the same level in the QD. This means that the phases at the wings of different resonances should coincide and the increase by $\pi$ at the resonance must be compensated. This compensation occurs via narrow jumps between the resonances and is accompanied by a fast rearrangement of the electrons in the dot.

Although the experiment [2] was clearly done in the $\mathrm{CB}$ regime, the widths of the resonances turned out to have been anomalously large, only few times smaller than the charging energy. Also the widths and heights of all observed resonances are very similar. These surprising features of the results of ref. [2], which have not attracted so wide an attention as the phase jumps, also find natural explanation within our picture. Our mechanism requires the QD not to be fully chaotic (neither do we require an integrable QD). It is not clear, how chaotic was the dot used in the experiment. However, the QD containing $\sim 200$ electrons was $\sim 50$ times smaller than the nominal elastic mean free path. Thus, disorder should not have been essential for the dynamics of the electrons.

It is generally believed that the $\mathrm{CB}$ is observed only if the widths of resonances are small compared to the single-particle level spacing in the dot $\Delta$. This condition assumes that couplings of all levels to the leads are of the same order of magnitude. However, as we will show at the end of this paper, even for nonintegrable ballistic QD-s the widths of the resonances may vary by orders of magnitude. In this case it does not make sense to compare the width of few broad resonances with the level spacing, determined by the majority of narrow, practically decoupled, levels.

A useful theoretical model for the description of charging effects in QD-s is the tunneling Hamiltonian in the constant interaction $\left(U_{C B}\right)$ approximation (see e.g. 四)

$$
\begin{aligned}
H & =\sum_{i} \varepsilon_{i} c_{i}^{+} c_{i}+U_{C B} \sum_{i<j} c_{i}^{+} c_{i} c_{j}^{+} c_{j} \\
& +\sum_{k} \varepsilon(k) a_{k}^{+} a_{k}+\sum_{k, i}\left[t_{i} c_{i}^{+} a_{k}+h . c .\right]
\end{aligned}
$$

Here $c\left(c^{+}\right)$and $a\left(a^{+}\right)$are the annihilation(creation) oper- 
ators for electrons in the dot and in the lead and $\varepsilon_{i}, \varepsilon(k)$ are the single-particle energies. We do not introduce the $k$ dependence of the tunneling matrix elements $t_{i}$. Since our approach is mainly based on the energetics of the QD, it is enough to consider only one lead. Summation over spin orientations is easily included. Also under the assumption of capacitive coupling to the gate, the levels in the dot flow uniformly with the voltage

$$
\varepsilon_{i}=\varepsilon_{i}\left(V_{g}=0\right)-V_{g} .
$$

The energies of the electrons in the wire are given by

$$
\varepsilon(k)=k^{2} / 2 m-E_{F} .
$$

Here $k=n \pi / L$, a (very)large $n$ is the level number in the wire and $L$ is the length of the wire.

For our purposes, it will be possible to simplify further the Hamiltonian (11). We will consider the case where the coupling of one particular level $N$ is dominant $t_{N} \gg$ $t_{i}, i \neq N$. If the width of this level is larger than the single-particle level spacing $\Delta$, a very nontrivial regime of charging of the QD may be described by means of secondorder perturbation theory estimates. Surprisingly, this simple limit of CB has not been considered yet.

An example of a system for which the widths differ drastically is the integrable QD [8,9]. However, it is hard to believe that the large $\left(N_{e} \sim 100 \div 1000\right)$ QD may be even close to integrable. Nevertheless, at least in classical mechanics, a considerable gap is left between integrable and fully chaotic systems. Even in a nonintegrable dot two kinds of trajectories - quasi-periodic and chaotic may coexist. In this case, in 2-dimensions any trajectory (even a chaotic one!) does not cover all the phase space allowed by energy conservation. Consequently, the corresponding wave functions do not cover all the area of the QD. If such a regime is realized in QD-s, it easily explains why the widths of the resonances may vary by orders of magnitude. Moreover, many other features of such a QD may differ strongly from those of the chaotic QD [17]. An explicit numerical example, which supports the existence of such regime will be given later.

Now we turn to the many-particle effects arising for the Hamiltonian (1) in the case of only one $(N$-th) level in the dot coupled strongly to the wire

$$
\Gamma \equiv \Gamma_{N}=2 \pi\left|t_{N}\right|^{2} d n / d \varepsilon \gg \Delta .
$$

(Here $n$ is the same as in the eq. (3) and $d n / d \varepsilon$ is taken at the Fermi energy $\varepsilon=0$.) The widths of the other levels are taken to be much smaller than the level spacing and may be neglected in the first approximation. The charging energy is still very large $U_{C B} \gg \Gamma$. We shall show that transmission of a current at about $(\Gamma / \Delta) \ln \left(U_{C B} / \Gamma\right)$ consecutive $\mathrm{CB}$ peaks will proceed through one and the same level $\varepsilon_{N}$.

Let the levels with $i \leq 0$ in the QD be occupied. Our aim is to find the total energy $E_{t o t}$ of the true ground state of the dot at different values of $V_{g}$. Without loss of generality we may assume that the summation over $i$ in eq. (1) goes only over $i>0$. (Thus we subtract from the total energy the trivial constant corresponding to selfinteraction of electrons with $i \leq 0$. Coulomb interaction between electrons at the levels with $i \leq 0$ and $i>0$ is included into $\varepsilon_{i>0}$.) Also let us subtract from the total energy the trivial energy of electron gas in the leads $\sum \varepsilon(k)$.

Let us consider spinless electrons. For large positive $\varepsilon_{N}\left(V_{g}\right) \gg \Gamma$ the only contribution to the total energy $E_{t o t}$ is given by the second order correction (the levels in the wire are lowered due to the repulsion from the unoccupied level $\varepsilon_{N}$ )

$$
E_{\text {tot }}^{(0)}=\int_{0}^{k_{F}} \frac{\left|t_{N}\right|^{2}}{\varepsilon(k)-\varepsilon_{N}} \frac{L}{\pi} d k=\frac{-\Gamma}{2 \pi} \ln \left(\frac{4 E_{F}}{\varepsilon_{N}}\right) .
$$

Here and everywhere below, $\varepsilon_{N}$ (as well as e.g. $\varepsilon_{1}$ ) is the function of $V_{g}$ (2). The upperscript (0) at $E_{\text {tot }}^{(0)}$ shows the number of electrons at the narrow levels (with $i>0$ ) in the QD. Generalization of this result for the case of negative $\varepsilon_{N}, \varepsilon_{N} \ll-\Gamma$ (the broad level being below the Fermi energy) is straightforward (note that the level $\varepsilon_{N}$ is occupied, not the level $\varepsilon_{1}$ as one might expect):

$$
E_{\text {tot }}^{(0)}=\varepsilon_{N}-\frac{\Gamma}{2 \pi} \ln \left(\frac{4 E_{F}}{\left|\varepsilon_{N}\right|}\right) .
$$

Here the first term $\varepsilon_{N}$ accounts for the energy loss due to replacement of one electron from lead to the dot. The second order level shift now includes both lowering of levels with $\varepsilon(k)<\varepsilon_{N}$ and raising of those with $\varepsilon(k)>\varepsilon_{N}$. The perturbative treatment fails for $\left|\varepsilon(k)-\varepsilon_{N}\right| \lesssim \Gamma$, but the corresponding shifts of levels below and above $\varepsilon_{N}$ evidently compensate each other, which is equivalent to taking the principal value of the integral in Eq (5). An approach related to ours was used recently in ref. [11] for the calculation of $\mathrm{CB}$ peaks positions.

Finally, the exact solution (for spinless electrons) for a single state interacting with a continuum is also known (e.g. [14]). A precise treatment of this situation, along the lines of ref. 15], yields:

$E_{\text {tot }}^{(0)}=\frac{-\Gamma}{4 \pi}\left[\ln \left(\frac{16 E_{F}^{2}}{\varepsilon_{N}^{2}+\Gamma^{2} / 4}\right)+2\right]+\frac{\varepsilon_{N}}{\pi} \cot ^{-1} \frac{2 \varepsilon_{N}}{\Gamma}$,

which coincides with the Eqs. (5.66) at $\left|\varepsilon_{N}\right| \gg \Gamma$.

Let us now consider the branch where the level 1 in the QD is occupied. The energy of this electron is $\varepsilon_{1}$. However, adding one more electron via the hopping $t_{N}$ now costs $\varepsilon_{N}+U_{C B}$. The ensuing reduction of the downward shift of the level $E_{t o t}^{(1)}$ is of crucial importance. The analog of Eq. (5) for $\varepsilon_{N}+U_{C B}>0$ now reads

$$
E_{\text {tot }}^{(1)}=\varepsilon_{1}-\frac{\Gamma}{2 \pi} \ln \left(\frac{4 E_{F}}{\varepsilon_{N}+U_{C B}}\right) .
$$


For small $V_{g}$ one has $E_{\text {tot }}^{(0)}<E_{\text {tot }}^{(1)}$ and the Eqs. (507,6) describe the true ground state of the system. However, the two functions $E_{\text {tot }}^{(0)}\left(V_{g}\right)$ and $E_{\text {tot }}^{(1)}\left(V_{g}\right)$ cross at

$$
\varepsilon_{N}=-\frac{U_{C B}}{\exp \left\{2 \pi\left(\varepsilon_{N}-\varepsilon_{1}\right) / \Gamma\right\}+1}
$$

and the ground state jumps onto the branch $E_{t o t}^{(1)}$. The energy of the current-transmitting virtual state $N$ is positive again. Thus, the transmission amplitude phase had returned to what it was before the process of filling of state $N$ and the subsequent sharp jump into the state where level 1 is filled. It is the latter jump which provides the sharp drop by $\pi$ of the transmission phase, following its increase by $\pi$ through the broad resonance. Many $\left(\sim(\Gamma / \Delta) \ln \left(U_{C B} / \Gamma\right)\right)$ consecutive resonances are due to the transition via one and the same level $N$.

For electrons with spin, the Breit-Wigner-related formula (7) does not work. However, far from the resonance the perturbation theory may still be used (at least until the temperature is high enough to be away from the Kondo effect [16]). We are not able to discuss in detail the role of spin in this short note. Still in this case the many charging events proceed via one and the same broad resonance, each accompanied by the increase of phase by $\pi$ which is compensated by the $-\pi$ jump in the valley.

To illustrate the relevance of the model eq. (1) with single strongly coupled level we performed numerical simulations for a model QD of a size $l$ with a simple polynomial potential (a smooth QD coupled to two leads)

$$
V=-4 x^{2}\left(1-\frac{x}{l}\right)^{2}+\left(y+\frac{x^{2}}{4 l}\right)^{2}\left(1+8\left(\frac{x}{l}-\frac{1}{2}\right)^{2}\right) \text {. }
$$

Due to the strong mixing of the $x$ and $y$ coordinates the dot is expected to be nonintegrable, but, similarly to the experimental geometry [2], it is approximately symmetric. For simulations we considered the $\mathrm{QD}$ on the lattice and used $l=10$ which was equivalent to 50 lattice spacings. The kinetic term is given by the standard nearest neighbor hopping. Below we present the results of calculations with the hopping matrix element $\tau=18$ which corresponds to the dot with $\sim 100$ electrons or $\sim 200$ if the spin is included (similar numbers to those in the experiment). We have used the potential $V$ of Eq.(10) for $0<x<l$. The lead formed by the potential $V=3 y^{2}$ was attached at $x<0$ and a hard wall at $x=l$.

Within the energy interval $1.5<\varepsilon<4.7$ only one mode may propagate along the lead. The analysis of solutions of the Schrödinger equation within this interval allowed us to find the positions and widths of quasi-stationary levels in the dot. As we expected, the widths fluctuate very strongly from level to level (by many orders of magnitude). In particular the widths of two levels \#102 and \#108 exceed sufficiently the level spacing $\Gamma / \Delta \approx 6$ (the number of states doubled due to spin). The widths of other levels vary from $\Gamma / \Delta \sim 1$ to $\Gamma / \Delta \sim 10^{-5}-10^{-6}$.
The origin of the hierarchy of widths becomes clear from fig. 1, where we have plotted $|\psi|^{2}$ in the QD for (real) $\varepsilon$ at the top of corresponding resonances. The quantized version of different variants of classical motion may be found on this figure. The most narrow level \#103 corresponds to a short stable transverse periodic orbit. Other broader levels, such as \#96,106, may be considered as the projections of the invariant tori corresponding to quasi-periodic classical motion. This classical trajectory reaches the line $V(x, y)=\varepsilon$ only at few points. The candidates for chaotic classical motion (e.g. \#110) also correspond to relatively broad resonances [12]. Even in this case only a part of the QD is covered by the trajectory. For the most coupled levels \#102 and \#108 the area covered by the trajectory touches the lead by its corner.

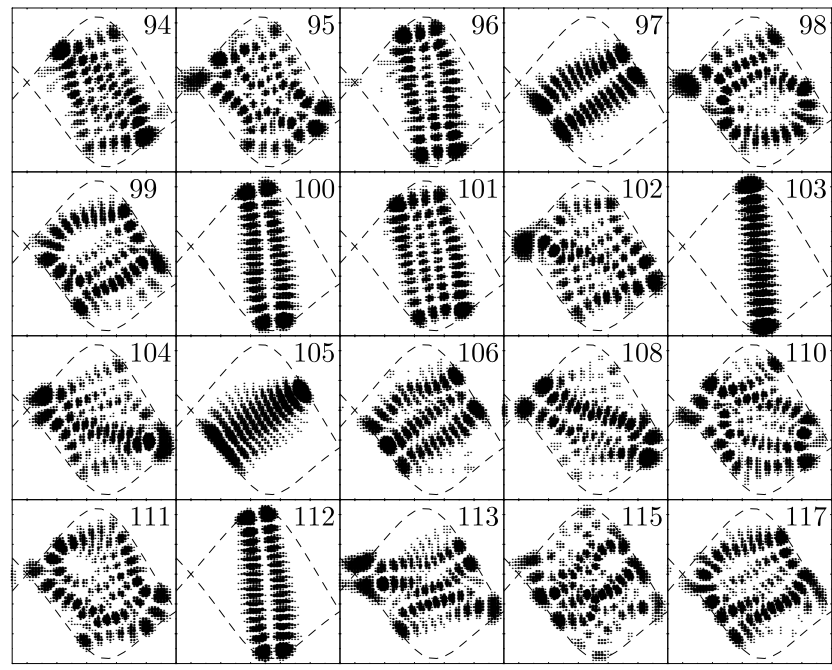

FIG. 1. The density of electrons in the dot at the resonances coupled to the single-channel wire(attached from the left). The line $V=0$ is shown dashed. Numbers correspond to the number of the level in the QD. About $95 \%$ of the norm of the wave function in the dot is shown. The "twin copies" (such as 100 and 112) of levels 94, 96, 101, 103 are not shown.

Moreover, two well coupled trajectories contribute to the level \#102. This is seen from the fig. 2 where we show also the $|\psi|^{2}$ at the left and right wings of this resonance. One contribution corresponds to the strongly coupled quasi-periodic trajectory (left), having the "turning point" $V(x, y)=\varepsilon$ just at the left contact. The other contribution comes from the true periodic trajectory (right). Two quantum states in the dot become mixed via interaction with the wire and form one broad (\#102) and one almost decoupled (\#104) resonance [13].

We have repeated the calculations several times for slightly different $V$ and in a broad range of variation of the hopping. Typically we saw the resonances of very different width and the origin of the most broad peaks was explained by simple classical arguments. 


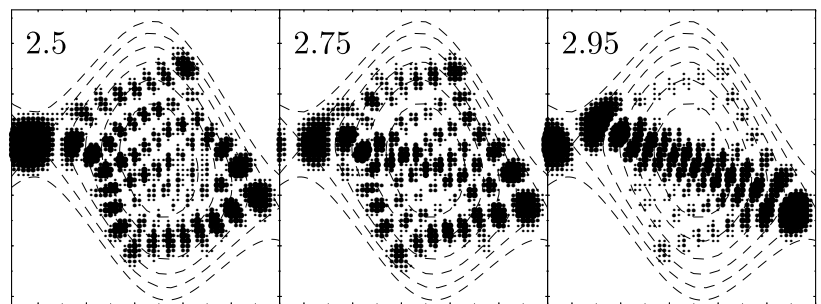

FIG. 2. Decomposition of the level 102 into parts corresponding to simple classical trajectories. Numbers are the energies for which the figures were done.

Taking into account the different sensitivities of longitudinal and transverse modes to the plunger [8,9] may allow to keep our broad level $\varepsilon_{N}$ even longer within the relevant strip of energy. This may provide an explanation of even longer sequences of resonances accompanied by the $-\pi$ jumps. In a more refined approach, adding new electrons into the QD should cause a change of the selfconsistent potential $V(x, y)$. The total energy of the dot and the wire will be lowered in the presence of strongly coupled levels. This may cause the potential of the QD to automatically adjust to allow such levels, which will support our explanation of the experiment of Ref. [2].

Our mechanism of charging of the QD requires the existence of the broad level with $\Gamma \gg \Delta$. The simple way to justify the relevance of our theory for the explanation of the experiment of ref. [2] will be to close the dot sufficiently in order to have $\Gamma \ll \Delta$ for all levels. In this case the phase still increases by $\pi$ at any resonance, but the correlation between peaks will disappear. (More precisely the pairs of peaks corresponding to adding of electrons with opposite spins onto one and the same level still are correlated, but correlation between pairs should disappear.) Moreover, within our mechanism a series of $\sim(\Gamma / \Delta) \ln \left(U_{C B} / \Gamma\right)$ strong charging peaks in the conductance should have the same height. This "coupling dependent" correlation of the peak heights seems also easy to measure.

To conclude, we have considered the model, for which upon increasing $V_{g}$, it is energetically favorable to first populate in the dot the level strongly coupled to the leads. At a somewhat larger $V_{g}$ a sharp jump occurs to a state where the "next in line" narrow level 1 becomes populated. This jump accounts for the sharp decrease by $\sim \pi$ of the transmission phase. The similar strengths of resonances seen in the experiment [2] and their large width are also clear within our mechanism. The current transmission through such a QD resembles the behavior of rare earth elements, whose chemical properties are determined not by the electrons with highest energy, but by the "strongly coupled" valence electrons. The overlapping of single-particle resonances may take place also in the Kondo experiments in QD-s [18], where in order to increase the Kondo temperature the dot is usually suffi- ciently opened. Hopefully the unusual effects observed in some of these experiments may be also explained within our approach.

Valuable discussions with E. Buks, M. Heiblum, Y. Gefen, I. Lerner, Y. Levinson, M. Schechter, V. V. Sokolov, D. Sprinzak, H. A. Weidenmuller and A. Yacoby are acknowledged. The work of PGS was supported by RFBR, grant 98-02-17905 . Work at WIS was supported by the Albert Einstein Minerva Center for Theoretical Physics and by grants from the GermanIsraeli Foundation (GIF), the Israel Science Foundation, Jerusalem and the Tekla and Simon Bond Fund for Submicron Electronics Research at the Weizmann Institute.

[1] L. P. Kouwenhoven et al., Mesosopic Electron Transport, Proceedings of the NATO ASI, edited by L. L. Sohn, L. P. Kouwenhoven and G. Schön (Kluwer 1997).

[2] E. Schuster et al., Nature, 385, 417 (1997).

[3] The two terminal setup of the early experiment (A. Yacoby et al., Phys. Rev. Lett., 74, 4047 (1995)) does not allow to measure the phase of transmission amplitude through a QD.

[4] See e.g. Single charge tunneling: Coulomb blockade phenomena in nanostructures, NATO ASI series, edited by H. Grabert and M. H. Devoret (Plenum press 1992).

[5] P.S.Deo and A.M.Jayannavar, Mod. Phys. Lett. 10, 787 (1996)

[6] H. Xu and W. Sheng, Phys. Rev. B 57, 11903 (1998); C.-M. Ryu and S. Y. Cho, Phys. Rev. B 58, 3572 (1998); H.-W. Lee, Phys. Rev. Lett. 82, 2358 (1999).

[7] Y. Oreg and Y. Gefen, Phys. Rev. B 55, 13726 (1997).

[8] G. Hackenbroich, W. D. Heiss and H. A. Weidenmüller, Phys. Rev. Lett. 79, 127 (1997).

[9] R. Baltin et al., Eur. Phys. J. B. 10, 119 (1999).

[10] R. Baltin and Y. Gefen, Phys. Rev. Lett. 83, 5094 (1999).

[11] A. Kaminski and L. I. Glazman, cond-mat/9911243.

[12] In order to find the convincing evidence of "chaos" one should consider smaller $\hbar$ (more electrons in the QD).

[13] V. V. Sokolov and V. G. Zelevinsky, Nucl. Phys. A504, 562 (1989).

[14] A. Bohr and B. R. Mottelson, Nuclear Structure, Benjamin, New York 1, 284 (1969).

[15] L.D. Landau and E.M. Lifschitz Quantum Mechanics, p. 555, Pergamon, Oxford (1976).

[16] L. I. Glazman and M. E. Raikh, JETP Lett. 47, 452 (1988); T. K. Ng and P. A. Lee, Phys. Rev. Lett. 61, 1768 (1988); N. S. Wingreen and Y. Meir, Phys. Rev. B 49, 11040 (1994).

[17] M. Stopa, Physica B 251, 228 (1998).

[18] D. Goldhaber-Gordon et al., Nature 391, 156 (1998); S. M. Cronenwett, T. H. Osterkamp, and L. P. Kouwenhoven, Science 281, 540 (1998); J. Schmid et al., Physica B 256-258, 182 (1998). 